\title{
La educación remota durante la pandemia, una nueva manifestación de violencia estructural y simbólica en México
}

\section{Distance education during the pandemic: a new manifestation of structural and symbolic violence in Mexico}

DOI: https://doi.org/10.32870/dse.v0i24.1064

\section{Rocío Jazmín Ávila Sánchez*}

\section{Resumen}

En México, la educación remota encontró una población en edad estudiantil con grandes desigualdades, así como un alto porcentaje de niñas, niños y adolescentes en situación de marginación y vulnerabilidad. El traslado de las violencias estructurales propias del ámbito escolar al territorio del hogar, visibilizó las brechas existentes y amplió la desigualdad, sumando precariedades y carencias a la vida de los menores que viven en zonas marginadas. Las aportaciones teóricas de Bourdieu respecto a la reproducción social y la violencia simbólica, así como de Galtung en torno a las violencias estructural, cultural y directa, permiten analizar desde otra faceta, distinta a la pedagógica, los efectos que el traslado de la educación presencial a la remota conlleva, así como los que tendrán en corto y mediano plazo. Con esta perspectiva, los resultados de logro académico, el acceso a internet y las características escolares se convierten en variables que nos permiten entender la reproducción de las desigualdades y el incremento de la violencia estructural en México.

Palabras claves: Violencia estructural - exclusión escolar - marginación - desigualdad educativa - educación remota.

\begin{abstract}
Distance education found in Mexico a student-age population with great inequalities, as well as a high percentage of children and teenage students in situations of marginalization and vulnerability. The transfer of structural violence typical of the school environment to the household made visible the existing gaps and widened inequality, adding precariousness and deprivation to the lives of minors living in marginalized areas. Bourdieu's theoretical contributions on social reproduction and symbolic violence as well as Galtung's on structural, cultural, and direct violence, allow us to analyze from another facet, different from the pedagogical one, the effects that the transfer of face-to-face education to the distance mode entails,
\end{abstract}

* Doctora en Teoría Política, Teoría Democrática y Administración Pública. Candidata al SNI. Líneas de investigación: políticas sectoriales en educación y desigualdad educativa. Responsable técnica de la Red de Investigadores para la Calidad de la Educación, Secretaría de Educación Pública. Tamaulipas. México. rociojavila28@gmail.com 
as well as those that they will have in the short and medium term. Under this perspective, the results of academic achievement, internet access and school characteristics become variables that help us to understand the reproduction of inequalities and the increase in structural violence in Mexico.

Key words: Structural violence - school exclusion - marginalization - educational inequality - remote education.

\section{Introducción}

En 1951, Isaac Asimov publicó un cuento llamado ¡Cómo se divertían! (The fun they had), en el que se plantea un escenario donde niñas y niños son instruidos por computadoras de manera individual, situados frente a una pantalla, evaluados de manera remota y sin compartir el proceso de enseñanza con otros compañeros... No tuvimos que esperar hasta 2157, año en el que se ubica la narrativa, para asistir a un escenario educativo que tiene algo de irreal. La suspensión de clases presenciales como una política sanitaria para disminuir el contagio del virus SARS-CoV-2, trajo consigo efectos adversos en todos los niveles educativos, los cuales han evidenciado la profunda brecha de desigualdad prexistente al afectar en mayor medida a la población con altos índices de marginación y algún tipo de vulnerabilidad, convirtiéndose en una limitante para el desarrollo de las potencialidades de los estudiantes.

Refiriéndonos al caso de México, la educación remota se encontró con una población en edad estudiantil con grandes desigualdades, así como con un alto porcentaje de niñas y niños en situación de marginación y vulnerabilidad. El traslado de las violencias estructurales propias del ámbito escolar al territorio del hogar visibilizó las brechas existentes y amplió la desigualdad, sumando precariedades y carencias a la vida de los menores que viven en zonas marginadas. Información proporcionada por el Fondo de las Naciones Unidas para la Infancia (Unicef, por sus siglas en inglés) señalan que en América Latina y el Caribe la repercusión de la pandemia en el desarrollo de la infancia ha sido mayor debido al número de días que han permanecido cerradas las escuelas, superior al de otros países, entre los que México encabeza la lista con más de 200 días sin clases presenciales, contados hasta el mes de marzo de 2021 (Unicef, 2021).

La misma fuente reporta que tres de cada cinco infantes que perdieron el año escolar viven en América Latina y el Caribe (Unicef, 2021). En este sentido, habrá que esperar a que se reanuden las clases presenciales para conocer el efecto que la suspensión tendrá en la deserción escolar, ya que la normativa de la Secretaría de Educación Pública impide dar de baja o reprobar a los alumnos que estaban inscritos en el ciclo escolar 2019-2020, aunque estos no mantengan contacto con sus docentes.

Profundizando en el caso de México, previo a la pandemia, las pruebas estandarizadas ya señalaban que un alto porcentaje de niñas, niños y adolescentes no alcanzaban los conocimientos esperados en matemáticas, ni en lenguaje y comunicación, además de mostrar las grandes 
brechas existentes donde los colectivos que habitan zonas rurales o de pobreza obtienen los indicadores más bajos (INEE, 2019).

Aunado al déficit educativo que afectará la adquisición de conocimientos, la población escolar ha visto disminuida la interacción social, el desarrollo emocional inherente a la convivencia y el acceso a programas de bienestar relacionados con la escuela, como el caso de los desayunos escolares que proveen, en muchos casos, el único alimento diario a los menores que asisten a la escuela. Estas carencias pueden considerarse como un aumento en la violencia estructural (Galtung, 1995, 1995b) que enfrenta la población en edad escolar con alto grado de marginación.

Estadísticamente se ha comprobado que la suma de precariedades durante la infancia incide directamente en el descenso de la movilidad social, reproduciendo condiciones de pobreza en la edad adulta ya que la marginación durante la infancia parece ser predictiva de una vida precaria en un porcentaje altamente significativo (Coneval, 2018b; Sandoval, 2008; Sobolewski, Amato, 2005), además de estar asociada con el incremento en la violencia (López-Santiago et al., 2017). Desde esta lógica, las consecuencias que la pandemia tendrá a mediano y largo plazo en la conformación social y en la ampliación de las brechas de desigualdad representarán un retroceso en el desarrollo económico y social de países como México (Coneval, 2019, 2019b).

Siguiendo la argumentación de Galtung (1995b), la violencia se interpreta en relación con el no desarrollo de las posibilidades potenciales del ser humano, de manera que cuando no se satisfacen las necesidades básicas, entre las que la educación ocupa un sitio relevante, se puede hablar de una forma de violencia estructural. Asimismo, el autor establece una relación profunda entre los tres tipos de violencia: estructural, cultural y directa, precisando que la violencia puede empezar en cualquiera de los polos, afirmación que cobra relevancia en países como México, que tiene altos indicadores de violencia directa.

A la información estadística acerca del logro educativo de los alumnos en México y los datos sobre acceso a internet recabados a partir de la Encuesta Nacional sobre Disponibilidad y Uso de Tecnologías de la Información en los Hogares (Inegi, 2020), se suman los indicadores sobre los efectos que la pandemia ha tenido en la economía familiar, definiendo un panorama poco halagüeño para el desempeño escolar de amplios sectores de la población en un corto plazo. Asimismo, la escasa audiencia de las clases "Aprende en Casa", que fue diseñada como la estrategia principal de la Secretaría de Educación Pública para mantener el aprendizaje de los alumnos de educación básica y que, en los hechos, se ha sustituido por el contacto docente-alumno realizado principalmente a través de aplicaciones como WhatsApp, brindan elementos para reflexionar sobre los nuevos factores que se suman a la violencia estructural prexistente.

Desde la perspectiva teórica de Galtung, las carencias que limitan el desarrollo de las potencialidades de los seres humanos son una manifestación de violencia, estructural que estará 
relacionada con las otras formas de violencia de la misma manera que se conectan los vértices de un triángulo; por otra parte, desde un enfoque derivado de la teoría de la reproducción de Bourdieu, se puede afirmar que la tasa de éxito escolar estará estrechamente relacionada con los capitales del individuo, por lo que, a mayor precariedad menor logro académico, lo que constituye una forma de violencia simbólica. En este caso el adjetivo simbólico reconoce implícitamente la existencia de otras manifestaciones de violencia, pero sin referirse a ellas de manera específica, lo que marca una diferencia con la articulación teórica de Galtung.

Referirse al concepto de violencia simbólica desde la perspectiva de Bourdieu, conlleva hablar de la escuela como institución y como espacio donde se reproducen las desigualdades con mecanismos implícitos, desde las acciones pedagógicas de docentes, directivos e instituciones sobre los educandos hasta el modelo de enseñanza definido por la clase/cultura que ocupa una posición de poder en la estructura social. Constituye, por lo tanto, una violencia dulce, invisible, que viene ejercida con el consenso de quien la padece y que esconde las relaciones de fuerza que están debajo de la relación en la que se configura (Bourdieu, 1994), por tanto, tiene más posibilidades de imponerse como forma de ejercer la dominación y la explotación. En consecuencia, el término está relacionado con el concepto de poder simbólico, es decir, con la capacidad que se tiene para imponer la visión legítima del mundo social, así como los medios para comprender y adaptarse al mundo social mediante un sentido común que representa de modo disfrazado el poder económico y político (Fernández, 2005).

De esta manera, a partir de la interpretación de las perspectivas teóricas de ambos autores es posible inferir que la educación remota instrumentada durante la pandemia ha sumado factores de exclusión, como la dificultad en el acceso a internet y la disponibilidad de equipos de cómputo, elementos que se constituyen en variables para analizar las profundas brechas tecnológicas existentes en las comunidades escolares en México.

La evidencia empírica permite observar las brechas de desigualdad escolar en alumnos de distintos estratos y que asisten a diferentes modalidades escolares (INEE, 2019); estas cifras indican una notoria disparidad entre los conocimientos manifestados en pruebas estandarizadas por diversos colectivos, mostrando un sesgo favorable para los grupos con mejores niveles de bienestar social. En este sentido, el análisis de datos estadísticos provenientes de fuentes oficiales respecto al logro educativo, al acceso a internet y al uso de plataformas sociales con fines educativos, permite obtener un panorama general a través del cual se busca establecer una relación entre la violencia estructural y la precariedad elevada por los efectos de la emergencia sanitaria, así como comprender los efectos de la contingencia en lo que se refiere a la operación pedagógica, las condiciones de vulnerabilidad y marginación. Con este objetivo, a partir de un enfoque metodológico mixto, se recurre a datos de la última década para establecer tendencias en cuanto a la vinculación de variables como educación y violencia, que se complementan con cifras presentadas de manera sincrónica para mostrar la situación de acceso y consumo de internet durante la pandemia. 


\section{Educación, desigualdad y marginación}

Las desigualdades educativas que se han evidenciado e incrementado durante el confinamiento, y la implementación de la educación remota, reflejan no sólo diferencias educativas sustantivas sino que resaltan la distribución desigual de capitales económicos, sociales y culturales (Bourdieu, Passeron, 1981, 2003), esto es en términos sistémicos, más que de capacidades individuales. Desde esta lógica, el acceso al conocimiento está determinado por cuestiones estructurales cuyas diferencias tienden a profundizarse y frente a las que el sujeto tiene limitadas posibilidades de acción.

Estudios recientes elaborados en el marco de la pandemia (Concheiro, 2020; Silas-Casillas, Vázquez, 2020), alertan sobre el profundo efecto que la educación remota tendrá a mediano y largo plazo en la condición de los estudiantes del sistema de educación superior en México, no sólo en el sentido pedagógico sino también en lo que refiere a los procesos de socialización necesarios para la consolidación de la vida comunitaria.

Aunque es indudable la afectación que el traslado de la educación presencial a la remota implica para los estudiantes de educación superior, y sobre todo para aquellos en condiciones de precariedad, la vulnerabilidad de la población infantil incrementa los efectos adversos de la suspensión de clases presenciales al sumar la dependencia que tienen los menores en aspectos esenciales de la vida cotidiana. Según datos del Coneval (2019b), la pobreza aumenta conforme la edad disminuye, siendo la población menor de 18 años en pobreza extrema la que tiene mayor cantidad de registros, seguidos por los adolescentes-jóvenes y los mayores de 65 años. ${ }^{1}$

Debe subrayarse que la educación constituye el eje primordial cuando hablamos de socialización ya que, además de contribuir al desarrollo integral de las personas en su contexto socioeconómico, a través de ella se forma y transforma a la sociedad, preparando a los futuros ciudadanos para su inserción en la vida pública. El sistema educativo es el agente principal que construye a los sujetos para la vida comunitaria, al proporcionar las herramientas para la formación integral propicia el acceso equitativo y diseña constantemente estrategias que abonen a la igualdad de oportunidades. De la elección y la implementación del modelo educativo dependerá en gran medida el resultado: la reproducción de las desigualdades y las violencias o la movilidad y el desarrollo social.

En este sentido, la evidencia empírica contradice los principios normativos. La necesidad de trasladar la enseñanza presencial hacia un modelo a distancia, ha provocado la ampliación de la brecha en el acceso a la educación y en los grados de aprendizajes. Las comunidades rurales, que históricamente han mostrado resultados académicos por debajo de la media como consecuencia de múltiples factores que van desde la estructura económica y social hasta el limitado acceso a las Tecnologías de la Información y la Comunicación Educativas (TICE), son

1 Para 2018 la población en pobreza es de $49.6 \%$ en menores de 18 años; de $42.4 \%$ en adolescentes y jóvenes menores de 29 años y de $41.1 \%$ en mayores de 65 años (Coneval, 2019b). 
también las que más han resentido el impacto negativo de la educación remota, lo que en los hechos agrava el riesgo de experimentar un retroceso en el aprendizaje, la eficiencia terminal, la permanencia y la tasa de absorción en las zonas rurales con indicadores elevados de marginación (tabla 1).

Tabla 1. Niñas, niños y adolescentes de o a 17 años en pobreza, 2015

\begin{tabular}{|l|c|c|}
\cline { 2 - 3 } \multicolumn{1}{c|}{} & Porcentaje de NNA en pobreza & Porcentaje de NNA en pobreza extrema \\
\hline Zonas urbanas & 41.7 & 4.5 \\
\hline Zonas rurales & 66.7 & 17.9 \\
\hline
\end{tabular}

Fuente: Coneval, 2019, con datos de la Encuesta Intercensal (2015).

La pobreza es un fenómeno que ha acompañado el desarrollo de las ciudades y a la que se ha tratado de combatir a través de programas institucionales y políticas sectoriales compensatorias que tienen como meta disminuir la brecha de desigualdad; sin embargo, como apuntan Bonal y Taravini (2010), las propuestas educativas para superar las situaciones de pobreza y exclusión son insuficientes si no se parte de que las condiciones materiales y sociales de vida son determinantes en el desarrollo social y, fundamentalmente, si además no existe un compromiso social para ello. En este sentido, es pertinente recordar el planteamiento de Bauman (2005), quien considera que una de las características del sistema posmoderno actual es el reconocimiento y naturalización de individuos residuales, para los que no se considera "útil" la implementación de políticas públicas porque no son remunerativas bajo el esquema de costobeneficio. Desde esta perspectiva, los programas sociales sólo tendrán como objetivo contener a las clases marginadas.

Asimismo, la educación, con su naturaleza eminentemente colectiva y sus fines emancipatorios es puesta en duda. La idea de éxito se basa en el mérito individual y en historias excepcionales que legitiman el fracaso de muchos, como consecuencia de la falta de capacidades y el esfuerzo insuficiente. Así, la escuela refuerza las características que le permiten reproducir, e incluso ampliar la desigualdad social, al internalizarla y por ende legitimarla (Bourdieu, 2000).

La reproducción de la desigualdad perpetúa la violencia estructural a la que se refiere Johan Galtung (1995), quien considera que la injusticia social, así como la distribución desigual del poder, es una forma de violencia estructural debido a que la desigualdad genera marginación y exclusión. Los indicadores publicados por el INEE respecto al logro educativo, analizado a través del Plan Nacional para la Evaluación de Aprendizajes (Planea), ilustran la brecha entre los resultados de las pruebas estandarizadas de conocimientos aplicadas en México (véase tabla 2 ), en los cuales las escuelas ubicadas en zonas de alta marginación obtienen resultados por debajo de aquellos que habitan zonas de baja marginación. 
Tabla 2. Resultado de Planea 2018 en alumnos de sexto grado, por tipo de escuela y grado de marginación

\begin{tabular}{|l|c|c|c|c|c|}
\hline Marginación & Promedio & General pública & indígenas & Comunitaria & Privada \\
\hline Muy Alta y Alta & 467 & 472 & 427 & 446 & 558 \\
\hline Media & 505 & 498 & 462 & 455 & 591 \\
\hline Baja y Muy Baja & 540 & 525 & 0 & 484 & 605 \\
\hline
\end{tabular}

Fuente: Elaboración propia con datos del INEE (2019).

Los cruces de variables demuestran la tendencia persistente de que la precariedad en aspectos económicos está asociada con el bajo desempeño académico; por ejemplo, en el caso de las comunidades indígenas, no existe ninguna escuela ubicada en zonas de baja marginación, y los resultados más altos se encuentran muy por debajo de los obtenidos en las escuelas de capital privado (véase tabla 2). Esto pareciera confirmar que, a poblaciones pobres, educación pobre.

Un reto pedagógico aun mayor es el que enfrentan las comunidades escolares de las escuelas multigrado, en las que un docente se hace cargo de todos los grados (escuelas unitarias) o de más de un grado (bidocentes o tridocentes). Los datos del INEE (2019) nos muestran una brecha en el logro académico de 43 puntos entre las escuelas multigrado y las de organización completa a nivel nacional. La dificultad de adaptar los contenidos diseñados para impartir clases de manera presencial al modelo remoto, en el caso de docentes de escuelas multigrado se eleva exponencialmente, sobre todo si consideramos que el mayor porcentaje de estas escuelas se ubica en contextos rurales de alta marginación y con una conectividad deficiente.

En estas condiciones, el cierre de las escuelas acrecienta el riesgo de abandono escolar, la carencia de herramientas que permitan la asistencia a clases remotas se suma a la reducción de ingresos que han experimentado $71 \%$ de los hogares con niñas, niños y adolescentes, según datos de Unicef (2019). Esta reducción de ingresos se traduce en que las familias den prioridad a gastos básicos y en los casos donde se pueda destinar algún tipo de recurso a la educación, se suele beneficiar a los hijos varones.

Esta privación del derecho a una educación de calidad es una forma de violencia estructural intrínsecamente relacionada con el incremento en la violencia directa, por ejemplo, Nateras y Zaragoza (2017) señalan que en México la deserción escolar es uno de los factores sociodemográficos determinantes de la delincuencia. Además, los datos estadísticos muestran una relación significativa entre delincuencia y bajo nivel de estudios (CIDE, 2012).

Para la década de 2010, una encuesta realizada por el CIDE (2012) mostró datos interesantes sobre el perfil de quienes se encuentran sentenciados por la comisión de delitos. Algunas cifras que cabe destacar relacionan pobreza, delincuencia y educación; por citar un ejemplo, se observa que el nivel educativo de quienes se encuentran presos está por debajo de la media 
nacional de escolaridad y más de $80 \%$ tienen estudios inferiores a secundaria (gráfico 1). Además, se menciona que la necesidad de trabajar es la causa principal para abandonar la escuela, lo que se asocia directamente con la pobreza; otro dato sugerente es que $10.5 \%$ de las mujeres declaran que dejaron de estudiar por presión de la familia, situación que no experimentó ningún hombre, así como también es significativamente mayor el número de mujeres que dejó de estudiar para casarse (véase tabla 3).

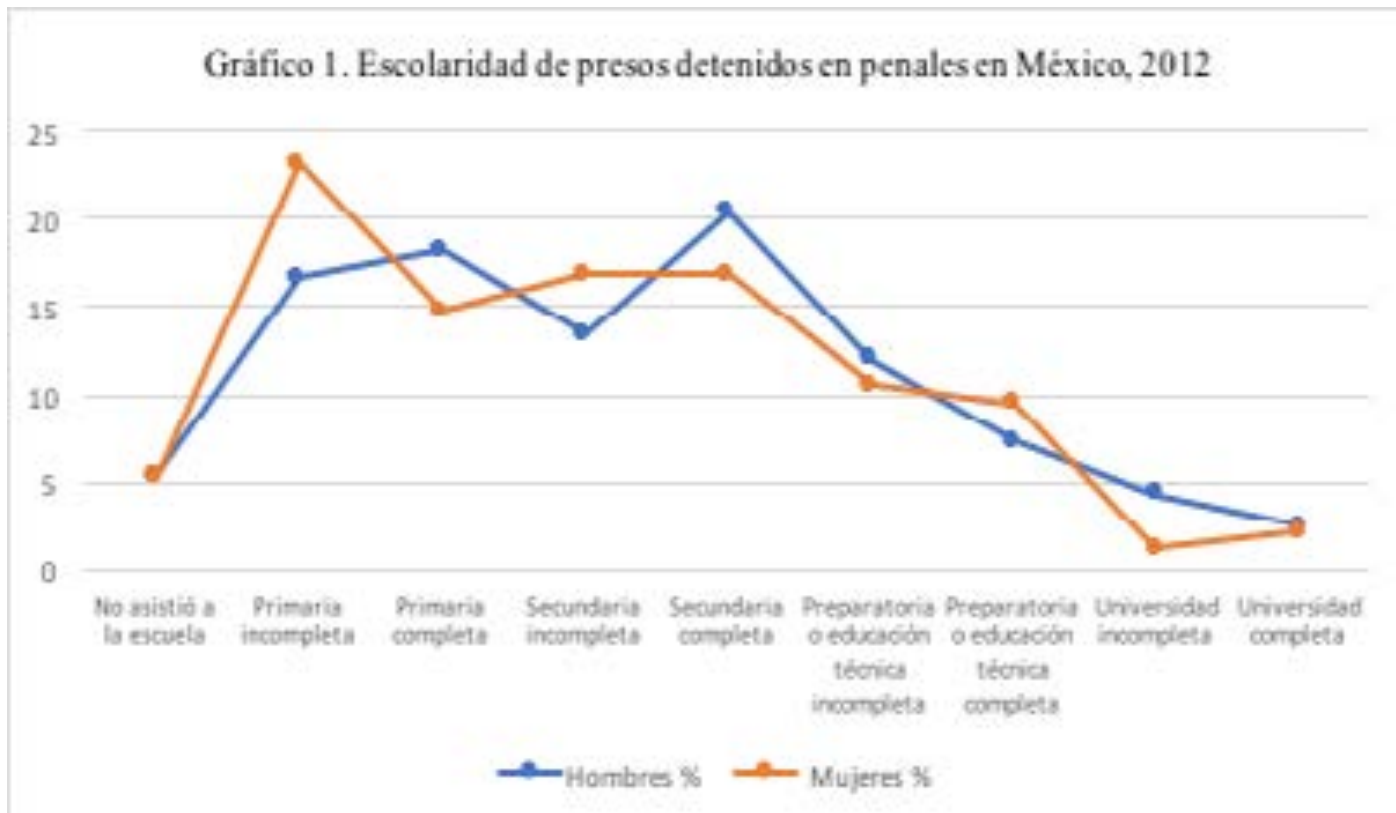

Fuente: elaboración propia con datos de la Primera Encuesta en Centros Penitenciarios Federales (CIDE, 2012).

Tabla 3. Principales causas de abandono escolar declaradas por presos que cumplían condenas en Centros Penitenciarios Federales en 2012

\begin{tabular}{|l|r|r|}
\hline Causa de abandono escolar & Hombres \% & Mujeres \% \\
\hline Necesidad de trabajar & 56.6 & 45.3 \\
\hline No le gustaba la escuela & 9.1 & 11.6 \\
\hline Falta de interés & 7.6 & 0 \\
\hline La familia le impidió seguir estudiando & 0 & 10.5 \\
\hline Abandonaron la escuela para casarse & 5.5 & 16.8 \\
\hline
\end{tabular}

Fuente: Elaboración propia con datos de la Primera Encuesta en Centros Penitenciarios Federales (CIDE, 2012).

En este sentido y siguiendo la reflexión de Galtung (1985), la pobreza mata a más personas que las guerras, pero ambas obstaculizan la autorrealización humana, en el sentido afectivo, somático y mental; además de ser generadoras de violencia. 


\section{Marginación tecnológica}

La desigualdad se va construyendo con diversos componentes y sus efectos son acumulativos. Antes de la puesta en marcha del modelo de educación remota, los indicadores sobre desempeño académico y los estudios de corte cuantitativo señalaban la amplia brecha que separaba a los alumnos con mejores puntajes de aquellos que ocupaban la parte baja de la tabla de resultados. La ubicación de la escuela, el turno y el tipo de organización son factores que inciden de manera directa en la apropiación del conocimiento.

La relación desigual de los individuos con la tecnología ha sido un tema recurrente desde antes de la pandemia; en algunos estudios se muestra que la brecha digital amplía y reproduce la inequidad social; en otros, que las nuevas tecnologías no resuelven el problema de los aprendizajes individuales y desempeños institucionales; algunos más, documentan un extendido escepticismo sobre las promesas de la educación virtual. Esos hallazgos parecen confirmarse con la experiencia de la pandemia (Acosta, 2020).

En México, para el año 2020 se registraban 80.6 millones de usuarios de internet, según datos obtenidos a partir de la Encuesta Nacional sobre Disponibilidad y Uso de Tecnologías de la Información en los Hogares (ENDUTIH), publicada por el INEGI en 2020. Aunque la cifra indica un incremento de 4.3 puntos porcentuales respecto a la de 2018, un análisis a profundidad muestra la desigualdad en el acceso al servicio de internet. La diferencia entre contextos rurales y urbanos es de 28.9 puntos porcentuales, lo que implica que más de la mitad de los habitantes en el ámbito rural no cuenta con servicio de internet (gráfico 2).

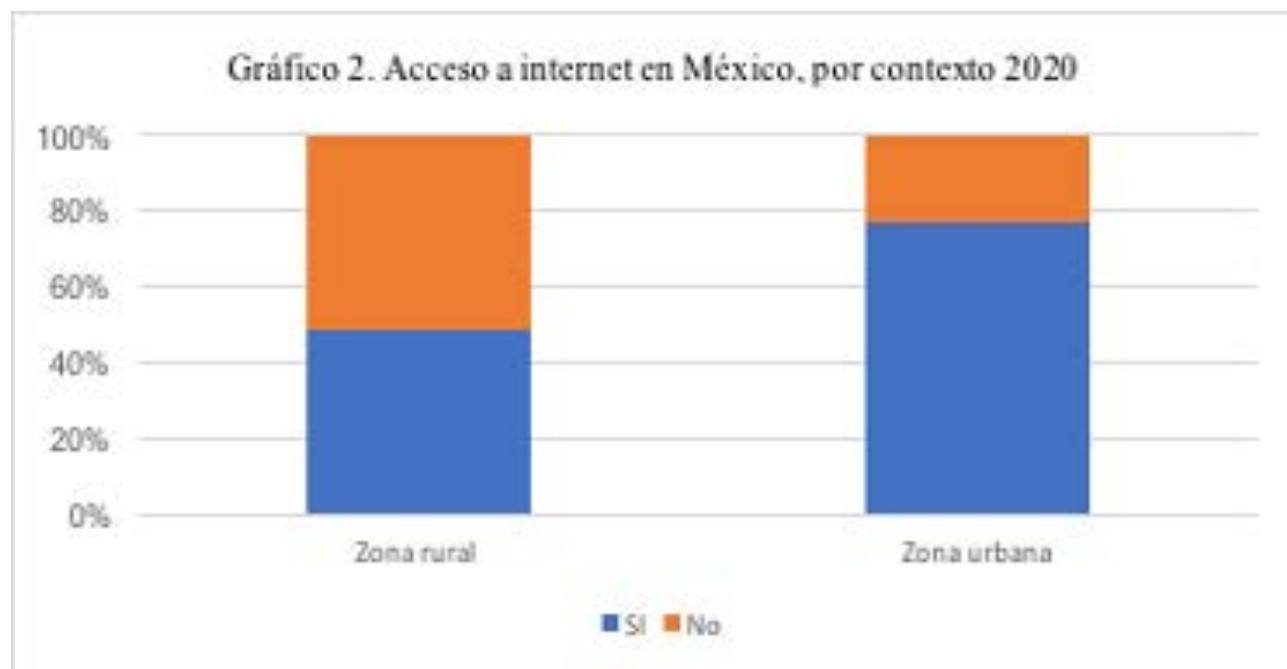

Fuente: ENDUTIH, INEGI (2020).

A esta brecha relacionada con el contexto, se suma la del nivel educativo ya que el número de usuarios que no cuenta con acceso a internet se incrementa en 37.3 puntos porcentuales 
cuando comparamos aquellos con nivel superior respecto de quienes tienen solamente educación básica (INEGl, 2020). Con lo cual la desigualdad de condiciones en alumnos que cursan estudios universitarios señalada por Concheiro (2020), aumenta para aquellos alumnos que cursan la educación básica en zonas rurales bajo la modalidad remota (gráfico 3).

\section{Grafico 3. Acceso a internet por nivel educativo. 2020}

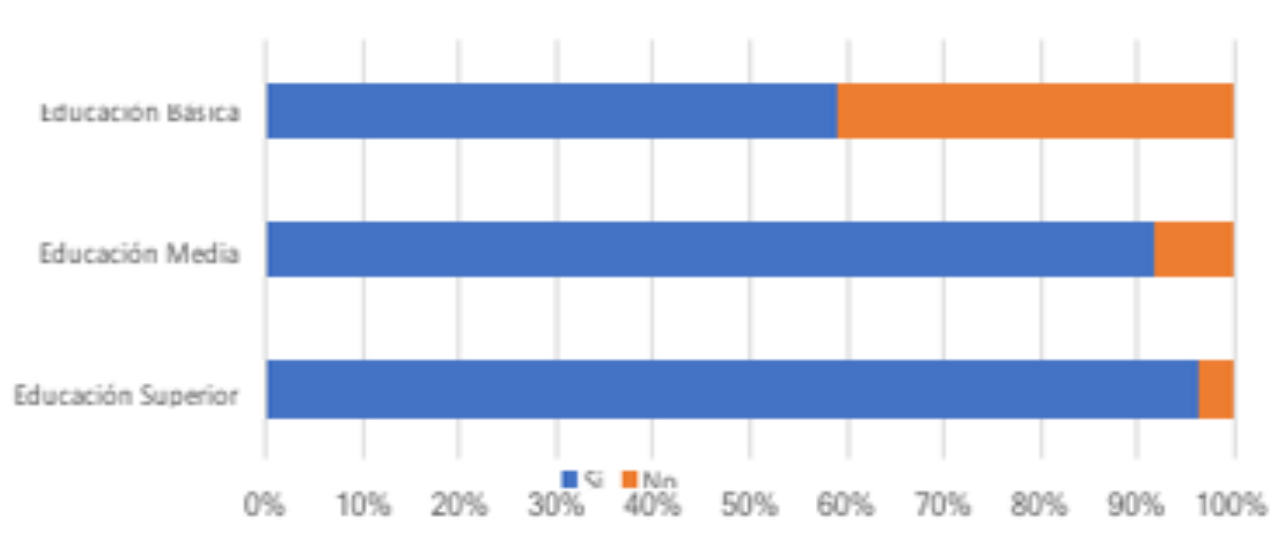

Fuente: ENDUTIH, INEGI (2020).

Desde un enfoque eminentemente pedagógico, el medio por el cual se accede a los servicios de internet es determinante. El proyecto de clases a distancia, diseñado por la Secretaría de Educación Pública (SEP) para impartir contenidos de manera remota a alumnos de educación básica, está diseñado para ser trasmitido a través de un televisor, en tanto la interacción entre docente y alumnos se deja al criterio de los participantes, bajo el supuesto de que se adaptará a las condiciones contextuales: geográficas, sociales y económicas.

Si bien las cifras a nivel nacional indican que $92.5 \%$ de la población tiene televisor, con una cobertura de casi $100 \%$ en zonas urbanas, en términos educativos el contacto se ha establecido principalmente a través de celulares y utilizando aplicaciones diseñadas con fines de diversión y no pedagógicos, cuyos costos de operación rebasan los ingresos de muchas familias y también de muchos docentes.

La migración de los componentes de los planes y programas desde el modelo presencial al formato en línea puso en evidencia muchos de los problemas que plagan al sistema educativo nacional (Lloyd, 2020), además de aportar datos que muestran que no todos los estudiantes están en condiciones de cumplir con sus deberes escolares, con lo que se puede observar otra faceta más de la inequidad histórica en el Sistema Educativo Mexicano (Silas, Vásquez, 2020).

Para ilustrar esta afirmación, se puede citar la encuesta de seguimiento académico Maestros contigo, realizada por la Secretaría de Educación de Tamaulipas (SET) a padres de familia de 
3,544 alumnos, en la que manifestaron que el contacto principal de los alumnos con el docente se da a través de la aplicación WhatsApp (gráfico 4). Incluso el incremento en el uso de teléfonos celulares inteligentes ${ }^{2}$ como medio para conectarse a internet, parece estar asociado con el descenso que se observa en el número de hogares que tienen una computadora, el cual bajó de $44.9 \%$ en 2018 a $44.3 \%$ en 2019 , siendo las computadoras portátiles más utilizadas que las de escritorio (INEGI, 2020).

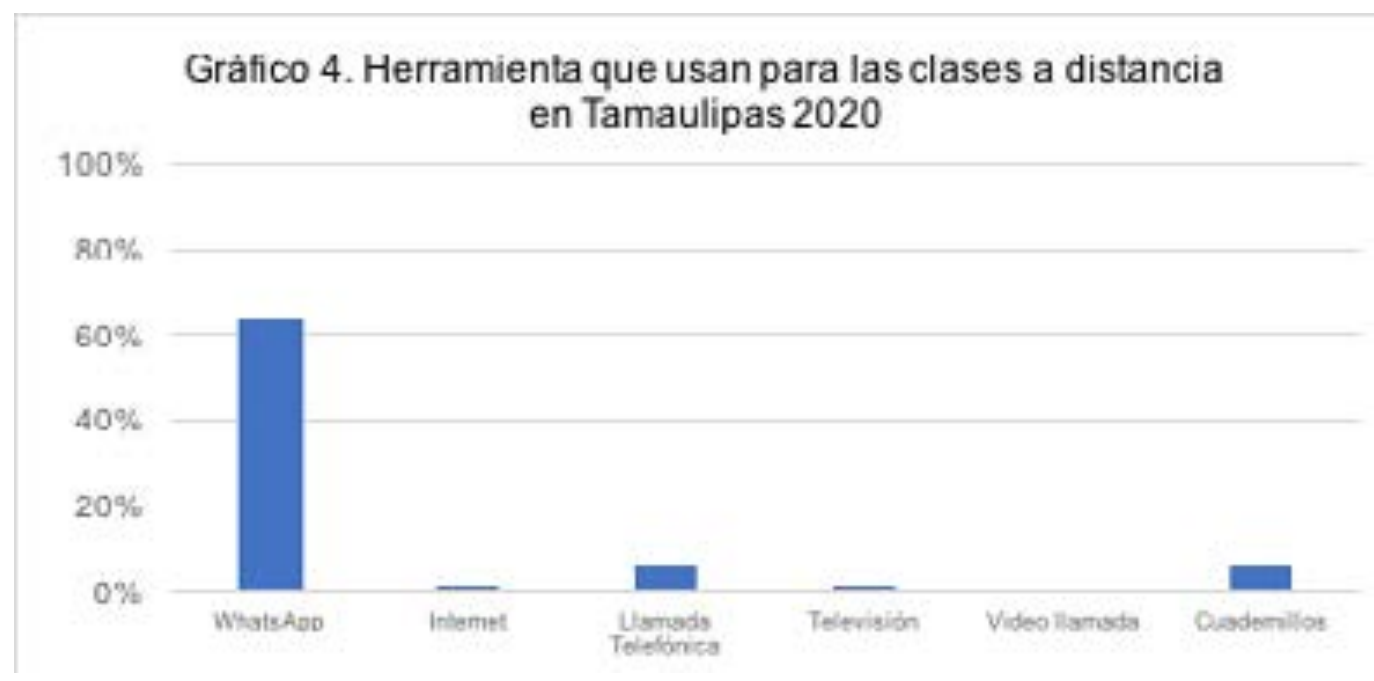

Fuente. Elaboración propia con datos de la Encuesta de seguimiento académico Maestros Contigo (SET, 2020).

\section{Conclusiones}

Siguiendo la reflexión de Concheiro (2020), que señala la marcada desigualdad que se observa en México y la consecuente ampliación de la brecha educativa que la pandemia implicará para la generación en edad escolar, es pertinente resaltar que en este país un total de 25 millones 253 mil 306 alumnos de educación básica se han visto afectados por el cierre temporal de sus escuelas, de los cuales algunos se incorporaron al modelo de educación remota desde sus hogares en "aulas sin paredes" (Bello, 2007). Otros - la mayoría - han visto precarizada la educación pública, e incluso han perdido acceso a programas compensatorios de alimentación que se materializaban en desayunos escolares y que representan una forma de garantizar la ingesta diaria de alimentos para niños en condiciones de marginación y pobreza.

Asimismo, las y los docentes, como actores esenciales del proceso educativo, también han sido afectados por el modelo de clases remotas, se han visto limitados en su esfuerzo por compensar las desigualdades de aprendizaje y generar aprendizajes colectivos, además de verse en la necesidad de pagar la conexión a internet con sus propios recursos.

2 De los usuarios, 95.3\% se conectan a internet a través de un celular inteligente (ENDUTIH, INEGI. 2020). 
Suárez y Ramírez-Stack (2020) afirman que es momento de pensar las metas educativas con perspectiva histórica y admitir que las carencias, la desigualdad y las inequidades que sufren millones de personas en México representan una amenaza para los logros sociales conquistados. Pero también para la estabilidad, la paz y el desarrollo, ya que toda manifestación de violencia directa tiene bases en la violencia estructural (Galtung).

Sabemos que la infraestructura de las escuelas ubicadas en zonas rurales tiene grandes deficiencias, 33\% de las escuelas no tiene red de agua potable y $72 \%$ no tiene drenaje; disparidades similares se observan entre las escuelas de municipios con rezago social bajo y muy bajo, así como alto y muy alto, donde las diferencias en las carencias de rubros como agua (13 y 41\%), drenaje (33 y 78\%) e internet (56 y 87\%) son importantes (INEE, 2019).

Simultáneamente, a las diferencias estructurales que privilegian a unos y violentan los derechos de otros, se construyen violencias simbólicas a través de las cuales se legitima la desigualdad y jerarquía. El aprendizaje social violento es un ejemplo cuando el currículum está basado en el individualismo y la competitividad, fragmentando el sentido de pertenencia a la comunidad. La educación impartida por los agentes dominantes que practican la acción pedagógica en estas comunidades ejerce un poder a través del cual se inculca el dominio jerárquico que busca mantener las relaciones de fuerza presentes en el sistema educativo nacional.

Todo suceso en la naturaleza humana y social está ligado a las circunstancias que lo provocan. Por ello, la homologación de las prácticas pedagógicas en detrimento de la implementación de políticas diseñadas con base en las necesidades de las comunidades marginadas incrementa las tasas de desigualdad, limita la movilidad social y refuerza la noción de jerarquía y autoridad.

Las reflexiones que se muestran a lo largo del documento representan el acercamiento a una línea temática de investigación que permitiría, a través del estudio de relaciones complejas, comprender los efectos de la violencia estructural (Galtung) y simbólica (Bourdieu) en las condiciones de vulnerabilidad y marginación que se derivan de los procesos educativos. Los efectos a corto plazo del confinamiento derivado de la emergencia sanitaria abren múltiples interrogantes que deberán ser analizadas desde perspectivas teóricas complementarias para comprender cómo se construyen las relaciones de dominación a través de la violencia simbólica que arranca sumisiones que ni siquiera se perciben como tales, apoyándose en unas expectativas colectivas y en unas creencias socialmente inculcadas.

Como en el cuento de Asimov, sería deseable reflexionar sobre el pasado, pero no con ánimo nostálgico, sino con el espíritu crítico necesario para generar el cambio imprescindible que se traduzca en mejores condiciones para todos, en el reconocimiento pleno de las diversidades, no con el afán de construir una sociedad homologada sino con el objetivo de reconocer en la alteridad la mejor arma para el desarrollo social, con equidad y justicia. 


\section{Referencias}

Acosta, A. (2020). ¿Aprender desde la crisis?: utopías, huellas y encrucijadas. México: COMIE.

Asimov, I. (1951). The fun they had.

Bello, R. (2007). Educación virtual: Aulas sin paredes. https://www.biblioteca.org.ar/libros-educar/10025.htm

Bonal, X.; A. Tarabini (2010). Ser pobre en la escuela. Habitus de pobreza y condiciones de educabilidad. Argentina: Miño y Dávila Editores.

Bourdieu, P. (1994). "La violenza simbólica". Entrevista Pierre Bourdieu. En la enciclopedia dell escienze filoso fiche de la RAl. Recuperado de: https://web.archive.org/web/20080513043250/ http://www.emsf.rai.it/interviste/interviste.asp?d=388

Bourdieu, P. (2000). La dominación masculina. Barcelona: Anagrama.

Bourdieu, P.; J. Passeron (1981). La reproducción: elementos para una teoría del sistema de enseñanza. Barcelona: Laia.

Bourdieu, P.; J. Passeron (2003). Los herederos: Ios estudiantes y la cultura. Buenos Aires: Siglo XXI.

CIDE (2012). Resultados de la Primera Encuesta realizada a Población Interna en Centros Federales de Readaptación Social. https://publiceconomics.files.wordpress.com/2013/01/encuesta internos cefereso 2012.pdf

Concheiro, F. L. (2020). Desafío del contexto previo a la pandemia y avances en la transformación de la Educación Superior en México. Revista Interdisciplinaria de Estudios Latinoamericanos, 4(3).

Coneval (2018). Medición de la pobreza. México: Coneval. https://www.coneval.org.mx/Medicion/Paginas/Pobrezalnicio.aspx

Coneval (2018b). Estudio Diagnóstico del Derecho a la Educación 2018. México: Coneval.

Coneval (2019) Pobreza infantil y adolescente en México 2008-2016. México: Coneval.

Coneval (2019b) Diez años de medición de pobreza multidimensional en México: avances y desafíos en política social. México: Coneval.

Fernández, J. (2005). La noción de violencia simbólica en la obra de Pierre Bourdieu: una aproximación crítica. (Cuadernos de Trabajo Social 18). España: Universidad Complutense de Madrid. Galtung, J. (1985). Sobre la paz. Barcelona: Fontamara.

Galtung, J. (1995). Investigaciones teóricas. Sociedad y cultura contemporáneas. Madrid: Tecnos / Instituto de Cultura Juan Gil-Albert.

Galtung, J. (1995b). Violencia, paz e investigación sobre la paz, en Investigaciones teóricas. Sociedad y cultura contemporáneas. Madrid: Tecnos.

INEE (2019). Evaluaciones de logro referidas al Sistema Educativo Nacional. Sexto Grado de Primaria. Ciclo escolar 2017-2018. México: INEE. https://www.inee.edu.mx/evaluaciones/planea/ sexto-primaria-ciclo-2017-2018/ 
INEE (2019b). Panorama educativo de México. Indicadores del Sistema Educativo Nacional 2018. Educación Básica y Media Superior. México: INEE. https://www.inee.edu.mx/wp-content/ uploads/2019/08/P1B117.pdf

INEGI (2020). Encuesta Nacional sobre Disponibilidad y Uso de Tecnologías de la Información en los Hogares (ENDUTIH). México: INEGI. https://www.inegi.org.mx/contenidos/saladeprensa/ aproposito/2020/eap internet20.pdf

López-Santiago, M.; M. Hernández-Juárez; A. León-Merino (2017). La marginación y exclusión como posibles factores socioeconómicos de la violencia urbana: el caso de Valle de Chalco Solidaridad, Estado de México. Papeles de Población, 23(91), 171-199. https://doi.org/10.22 $185 / 24487147.2017 .91 .008$

Lloyd, M. (2020). Desigualdades educativas y la brecha digital en tiempos de Covid-19. En Casanova, H. (coord.). Educación y pandemia. Una visión académica. México: IISUE-UNAM, 115121.

Nateras, M.; D. Zaragoza (2017). La pobreza como indicador de generación de la violencia y la delincuencia en México. En Betancourt, F. (coord.). Reflexiones sobre el estado de derecho, la seguridad pública y el desarrollo de México y América Latina. México: UNAM.

SET (2020). Encuesta de seguimiento académico Maestros Contigo. México: SET.

Sandoval, E. (2008). Estudios sobre pobreza, marginación y desigualdad en Monterrey. Estado de México. Papeles de Población, 14(57).

Silas-Casillas, J.; S. Vásquez-Rodríguez (2020). El docente universitario frente a las tensiones que le plantea la pandemia. Revista Latinoamericana de Estudios Educativos, 50, 89-120.

Sobolewski, J.; P. Amato (2005). Economic Hardship in the Family of Origin and Children's Psychological Well-Being in Adulthood. Journal of Marriage and Family, 67(1).

Suárez, M.; J. Martínez-Stack (2020). Covid-19. Efectos de la desigualdad social y la inequidad en la educación superior. Notas de Coyuntura del CRIM, (15). México: CRIM-UNAM.

UNICEF (2021). 3 de cada 5 niños y niñas que perdieron un año escolar en el mundo durante la pandemia viven en América Latina y el Caribe. México: UNICEF. https://www.unicef.org/ mexico/comunicados-prensa/3-de-cada-5-niños-y-niñas-que-perdieron-un-año-escolaren-el-mundo-durante-la 